\title{
Optimized active multicore fiber bending sensor
}

\author{
Juan A. Vallés ${ }^{\mathrm{a}, \mathrm{b}, *}$, David Benedicto ${ }^{\mathrm{a}}$ \\ ${ }^{a}$ Department of Applied Physics and I3A, Faculty of Sciences, University of Zaragoza, C/P. Cerbuna 12, 50009, Zaragoza, Spain \\ ${ }^{\mathrm{b}}$ Aragon Institute of Engineering Research, C/ Mariano Esquilor s/n, 50018, Zaragoza, Spain
}

A R T I C L E I N F O

\section{Keywords:}

Multicore waveguide

$\mathrm{Er}^{3+}$-doping

Modal interferometer

Bending sensor

\begin{abstract}
A B S T R A C T
We present a study of the feasibility of transforming the multicore fiber in a supermode-interference based bending sensor into an active stage. By compensating the antisymmetric supermode losses for high curvatures, fringe visibility is improved and the sensor operating range can be extended. For a given multicore fibre length and $\mathrm{Er}^{3+}$-ion concentration sensor calibration curves allow assessing the bending radius by measuring the visibility as a function of the input pump power. $\mathrm{The} \mathrm{Er}^{3+}$-ion concentration, available input pump power and multicore fiber length set the minimum bending radius that could be measured.
\end{abstract}

\section{Introduction}

The measurement of curvature radii is essential in structural monitoring applications such as in mechanical and aerospace engineering, and there are several types of optical fiber sensors well suited for these applications. Most of them are based on some kind of fiber gratings, like Bragg gratings or long-period gratings or on the Mach-Zehnder interferometer, where special structures like multicore fibers (MCF) have shown a good performance [1,2]. Fiber sensing systems based on MCF offer new possibilities to measure temperature, refractive index, strain as well as curvature, by taking advantage of coupling between adjacent cores that causes the so called supermodes to interfere during their propagation. This modal interference is very sensitive to external disturbances and can be used as a sensor.

An optical fiber bending sensor based on a 7-core hexagonal MCF modal interferometer uses the fundamental and antisymmetric supermodes (see Fig. 1), both of them excited by coupling a single mode fiber (SMF) to the central core of the MCF [3]. One way of assessing a curvature is to measure fringe visibility in the transmission spectrum and in order to achieve the optimal visibility, both supermodes must be evenly excited. However, when using the sensor for a small curvature radius, supermodes may have significantly different losses, and their interfering contributions become progressively imbalanced until one of the supermodes vanishes and accordingly modal interference. In this situation, if the MCF could be turned into an active stage with a tailored doping profile so that modal losses could be compensated, the restored visibility would improve the sensing performance and extend the curvature radius sensing range.

In this paper we explore this possibility by analyzing in section 2 the supermode imbalance caused by bending losses and determining the suitable erbium doping profile to neutralize this visibility reducing effect. Then, in section 3 the performance of the active MCF bending sensor is calculated as a function of the passive (MCF length) and active (doping level, input pump power) design parameters.

\section{Sensor design}

\subsection{Multicore interferometer passive design}

In waveguiding structures with multiple cores, the eigenmodes of the structure, so called supermodes, can be perceived combination of the individual modes of the single core waveguides. Supermodes are useful in order to understand the MCF properties [4], and have been directly utilized in optical communication systems [5]. The supermodes of the structures can be computed either through analytical methods or by numerical simulations [6]. In this study we have used a commercial software, called RSoft CAD, and its mode solver tool, FemSim, in order to obtain and characterize the supermodes of the hexagonal 7-core MCF.

The structure under study consists on a fiber with a central core and six more cores organized around it in a hexagonal pattern. The core diameter $(8.2 \mu \mathrm{m})$, together with the cladding and core refractive indices $\left(\mathrm{n}_{\text {clad }}=1.444, \Delta \mathrm{n}=0.003\right)$ and the diameter of the circle in which the hexagon is inscribed $(22 \mu \mathrm{m})$, have been chosen so that a standard SMF equally excites both the fundamental and antisymmetric supermodes. The amount of each mode excited by the single mode fiber, i.e. the normalized modal amplitude $a_{m}$ of the $m$-supermode, can be computed by using the expression [6]:

\footnotetext{
* Corresponding author. Department of Applied Physics and I3A, Faculty of Sciences, University of Zaragoza, C/P. Cerbuna 12, 50009, Zaragoza, Spain.

E-mail address: juanval@unizar.es (J.A. Vallés).
} 

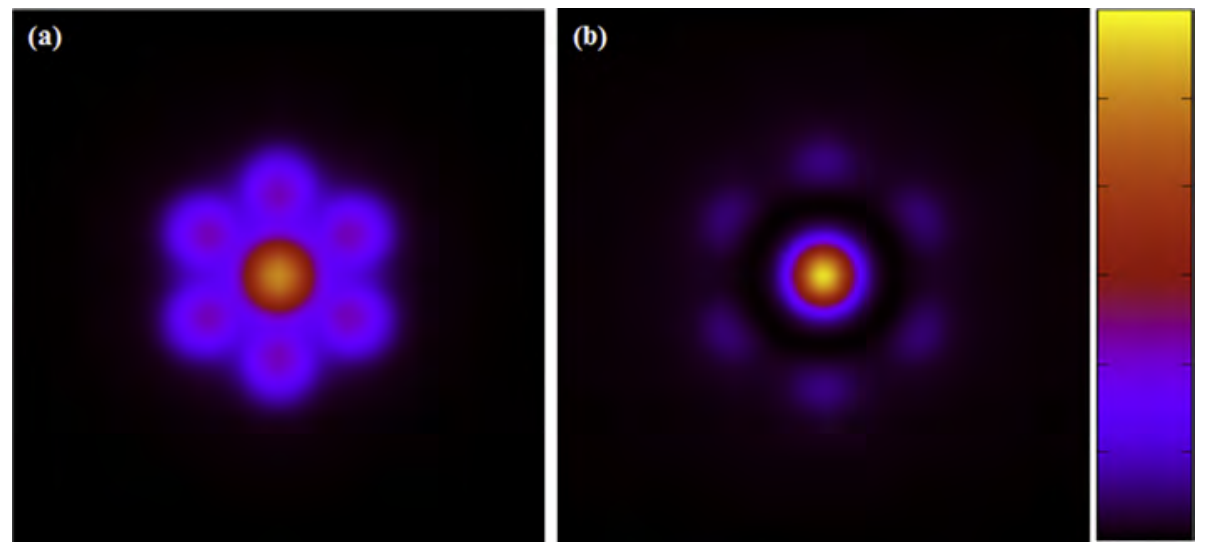

Fig. 1. (A) Fundamental and (b) antisymmetric computed transverse mode intensity profiles of a hexagonal 7-core MCF.

$a_{m}=\frac{\left|\int E_{m}(x, y) \cdot E(x, y) d A\right|^{2}}{\int\left|E_{m}(x, y)\right| d A \int|E(x, y)| d A}$,

where $E(x, y)$ and $E_{m}(x, y)$ are the field profiles of the excitation and of the $m$-supermode. As the modal amplitude depends on the field profiles, which depend on its turn on the wavelength, the selected geometrical parameters optimize the MCF performance for the maximum emission peak of the $\mathrm{Er}^{3+}$ ion, $\lambda=1534 \mathrm{~nm}$, because it is the wavelength at which the active device will operate.

\subsection{Bending losses}

Supermodes confinement is an important factor to take into account when studying curvature losses. As the fundamental ( $\mathrm{f}$ ) mode is more confined than the antisymmetric (a) one $\left(\mathrm{n}_{\text {eff,f }}>\mathrm{n}_{\text {eff,a }}\right)$, its bending losses can be expected to be lower. We compute supermode bending losses with the light propagation simulation tool BeamPROP, from the mentioned CAD software. Minimum operational curvature radii, i.e. maximum losses, of demonstrated MCF bending sensors are in the range of tens of cm: $54 \mathrm{~cm}$ [7], $23 \mathrm{~cm}$ [8] and $19 \mathrm{~cm} \mathrm{[3].}$

Fig. 2 shows the curvature losses of the antisymmetric supermode. As the curvature losses of the fundamental supermode are comparably negligible (three orders of magnitude smaller) we just consider those of the antisymmetric supermode. For bending radius below $13 \mathrm{~cm}$ bending losses reach values higher than some $\mathrm{dB} / \mathrm{cm}$ which may not be easily achievable with the amplifying stage gain.

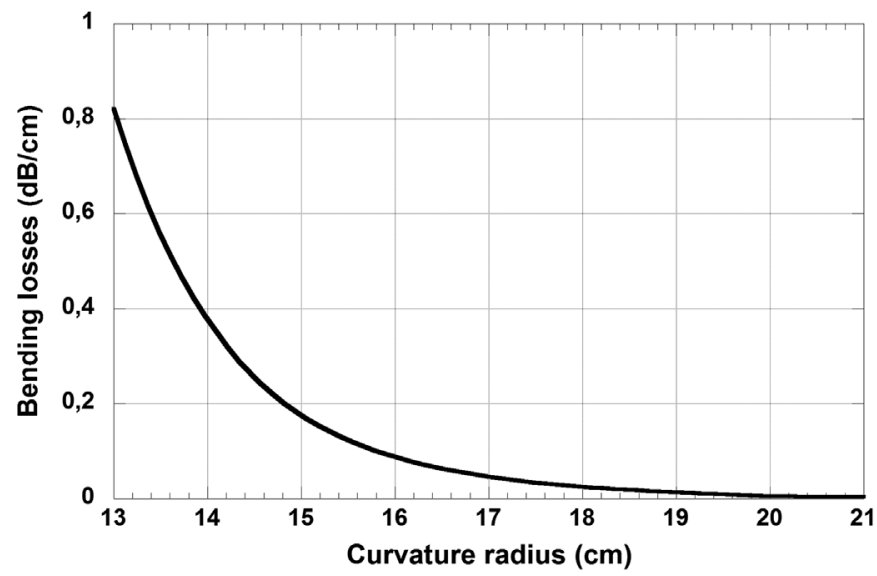

Fig. 2. Curvature losses for the antisymmetric supermode as a function of the curvature radius.

\subsection{Active stage}

Signal amplification depends on the overlap among its field profile, the pump field profile and on the doping profile. From the different possibilities in the doping transversal distribution, we study whether it is more convenient either to dope the central core or the external ones. As the overlap between the antisymmetric supermode and the center core is larger than that with the fundamental one, and the pump power is mainly transmitted along the central core (due to its shorter wavelength and higher confinement) the antisymmetric supermode achieves a higher amplification than the fundamental one. In order to achieve the desired mode power balance mode gain difference has to compensate the mode losses deviation. Since bending losses up to the $\mathrm{dB} / \mathrm{cm}$ range have to be compensated high erbium ion concentrations are needed, $\mathrm{n}_{\mathrm{Er}}=10^{25}$ ion $/ \mathrm{m}^{3}$ or higher. Moreover, the available pump power determines the active MCF required length. Fig. 3 shows the optical power difference between both supermodes as a function of the $\mathrm{Er}^{3+}$ ion concentration in the central core for $\mathrm{L}=10 \mathrm{~cm}$ and $\mathrm{P}_{\mathrm{p}}=315 \mathrm{~mW}$. We consider dopant concentrations up to $10^{26} \mathrm{ion} / \mathrm{m}^{3}$, which can be found in commercial fibers. With a high enough pump power (of hundreds of $\mathrm{mW}$ ) this concentration allows supermode power balance for bending radius over $13 \mathrm{~cm}$.

In order to compute each of the supermodes gain and optimize the interferometer design, we have used a home-made computer code which evaluates the set of power propagation equations of the supermodes coupled to the rate equations of the population densities of the active ions involved levels. The well-known models commonly used for step index fibers [9] can be used to describe the signal and pump

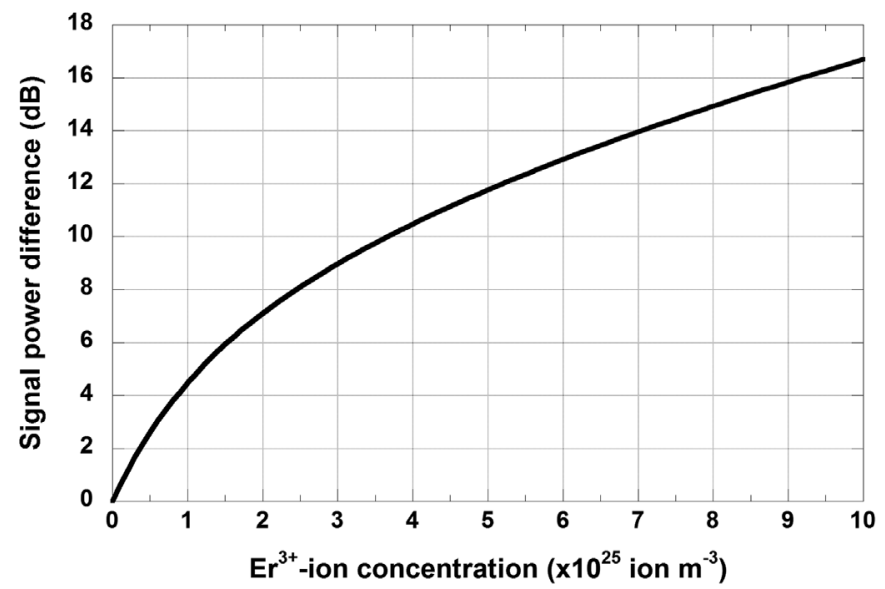

Fig. 3. Optical power difference between the antisymmetric and the fundamental supermodes as a function of the erbium ions concentration, defined as $\Delta=10 \cdot \log _{10}\left(\delta P_{n_{E r^{3+}}} / \delta P_{0}\right)$ for $\mathrm{L}=10 \mathrm{~cm}$ and $\mathrm{P}_{\mathrm{p}}=315 \mathrm{~mW}$. 


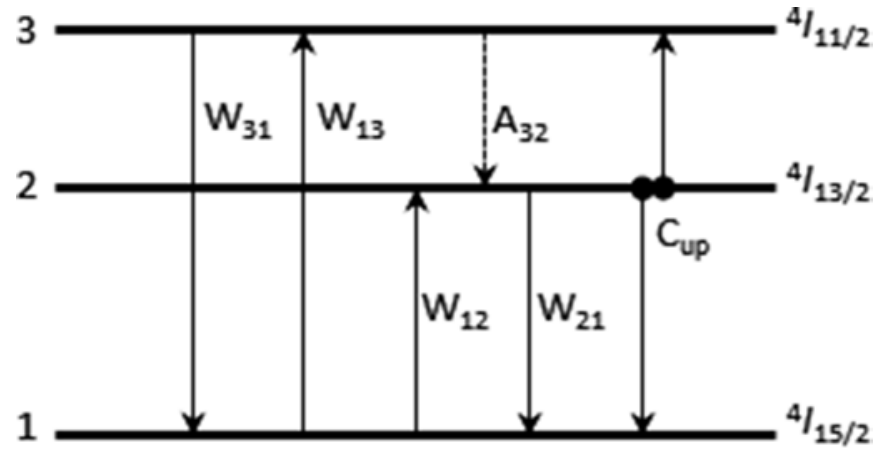

Fig. 4. Energy levels of $\mathrm{Er}^{3+}$ ion used in the model and the transition rates involved.

Table 1

Constructive supermodal interference lengths for $1534 \mathrm{~nm}$ and nearest destructive supermodal interference wavelengths for the three considered cases.

\begin{tabular}{lll}
\hline Length $(\mathrm{cm})$ & Transmission minima $(\mathrm{nm})$ & \\
\hline 1.07 & 1440 & 1635 \\
4.96 & 1514 & 1554 \\
9.92 & 1524 & 1544 \\
\hline
\end{tabular}
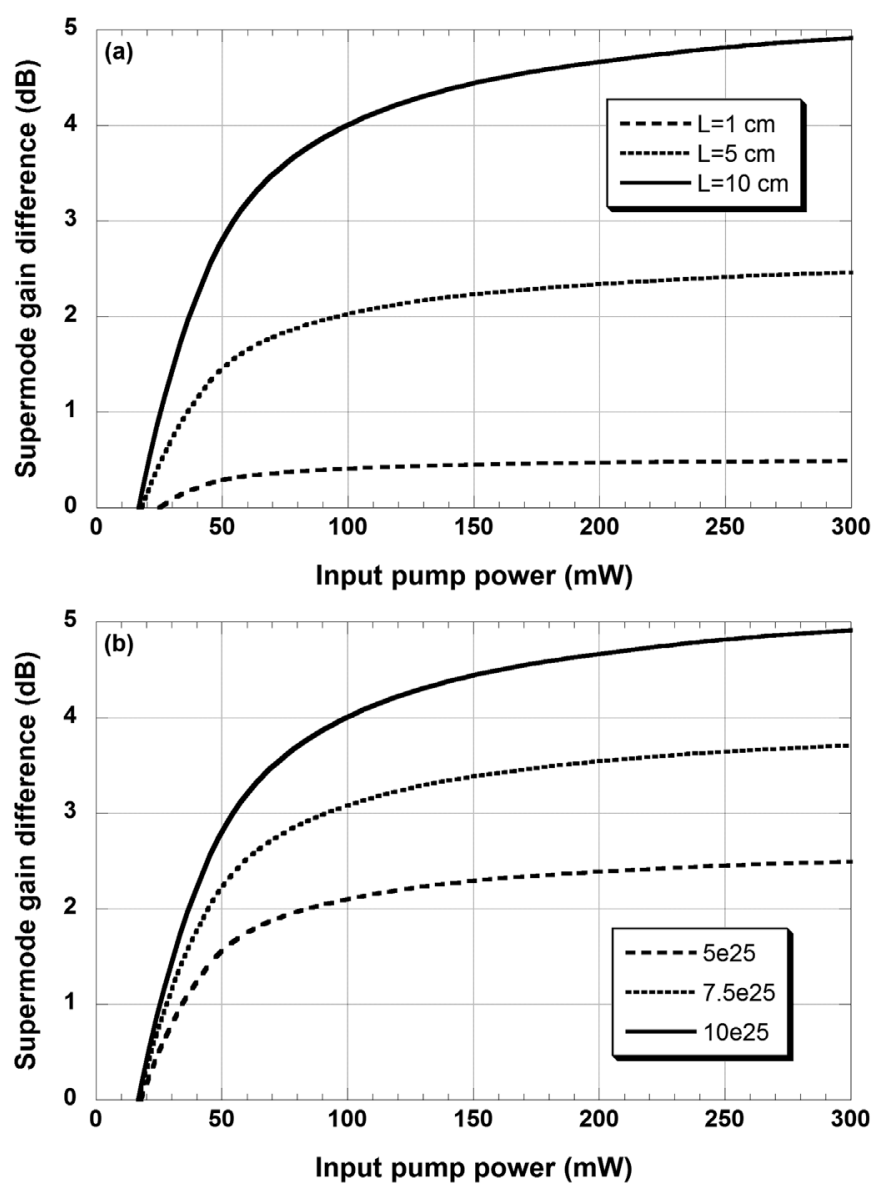

Fig. 5. Gain difference between the antisymmetric and the fundamental supermodes as a function of the pump power when the central core is doped and in the case of small signal. At the left (a) the gain difference is shown for three different lengths keeping a constant erbium concentration of $10^{26} \mathrm{ions} / \mathrm{m}^{3}$. At the right (b) the length is kept constant at $\mathrm{L}=10 \mathrm{~cm}$ in order to show three cases for different erbium concentrations.
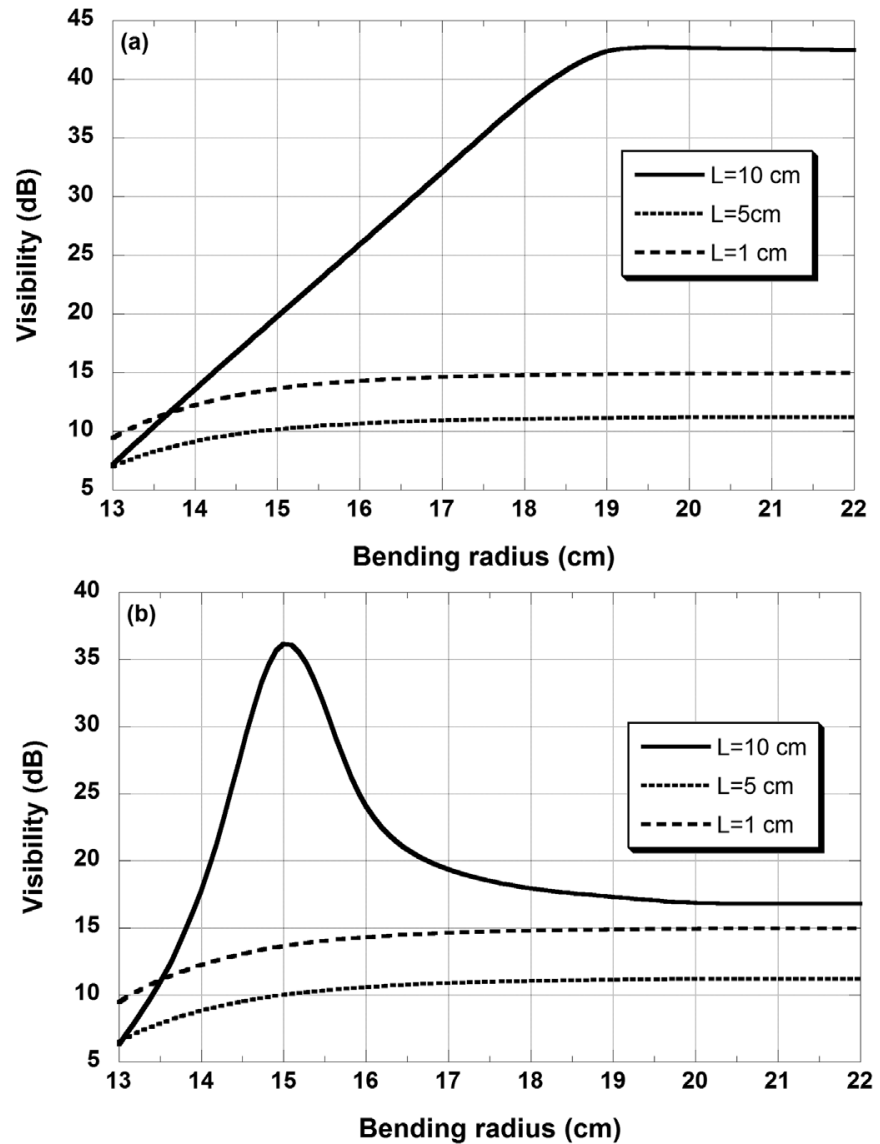

Fig. 6. Visibility as a function of the bending radius for three MCF lengths with respect to the nearest shorter wavelength minima (a) and higher wavelength minima (b).

evolution, together with the amplified spontaneous emission (ASE), along the erbium-doped MCF. As we are dealing with highly-doped waveguides, it is mandatory to take into account the upconversion process which has disadvantageous impact on the pump efficiency [10]. Fig. 4 shows a scheme of the energy levels and transitions, where $A_{i j}$ and $W_{i j}$ denote spontaneous and stimulated transition rates, respectively, and $C_{u p}$ represents the upconversion coefficient.

The population densities $n_{1}, n_{2}$ and $n_{3}$ of the energy levels ${ }^{4} I_{15 / 2}$, ${ }^{4} I_{13 / 2}$ and ${ }^{4} I_{11 / 2}$, respectively, are related by the equation $n_{1}+n_{2}+n_{3}=n_{E r}$, where $n_{E r}$ is the $\mathrm{Er}^{3+}$-ion concentration inside the central core, and equals zero outside. The temporal evolution of the population densities is given by the rate equations:

$\frac{d n_{1}}{d t}=-\left(W_{12}+W_{13}\right) n_{1}+\left(A_{21}+W_{21}\right) n_{2}+C_{u p} n_{2}^{2}$

$\frac{d n_{2}}{d t}=W_{12} n_{1}-\left(A_{21}+W_{21}\right) n_{2}+A_{32} n_{3}-2 C_{u p} n_{2}^{2}$

$\frac{d n_{3}}{d t}=W_{13} n_{1}-A_{32} n_{3}+C_{u p} n_{2}^{2}$

where $W_{12}, W_{21}, W_{13}$ and $W_{31}$ are the stimulated rates, proportional to the cross sections coefficients. The power propagation equations express the evolution of the optical power of the supermode $i, P_{\gamma, i}(\gamma=p$ for pump, $s$ for signal and $f$ for the amplified spontaneous emission), along the propagation axis and are given by Ref. [9]:

$\frac{d P_{p, i}^{ \pm}\left(z, v_{p}\right)}{d z}=\mp\left[N_{1, p, i}(z) \sigma_{13}\left(v_{p}\right)+\alpha_{p, i}\right] P_{p, i}^{ \pm}\left(z, v_{p}\right)$ 

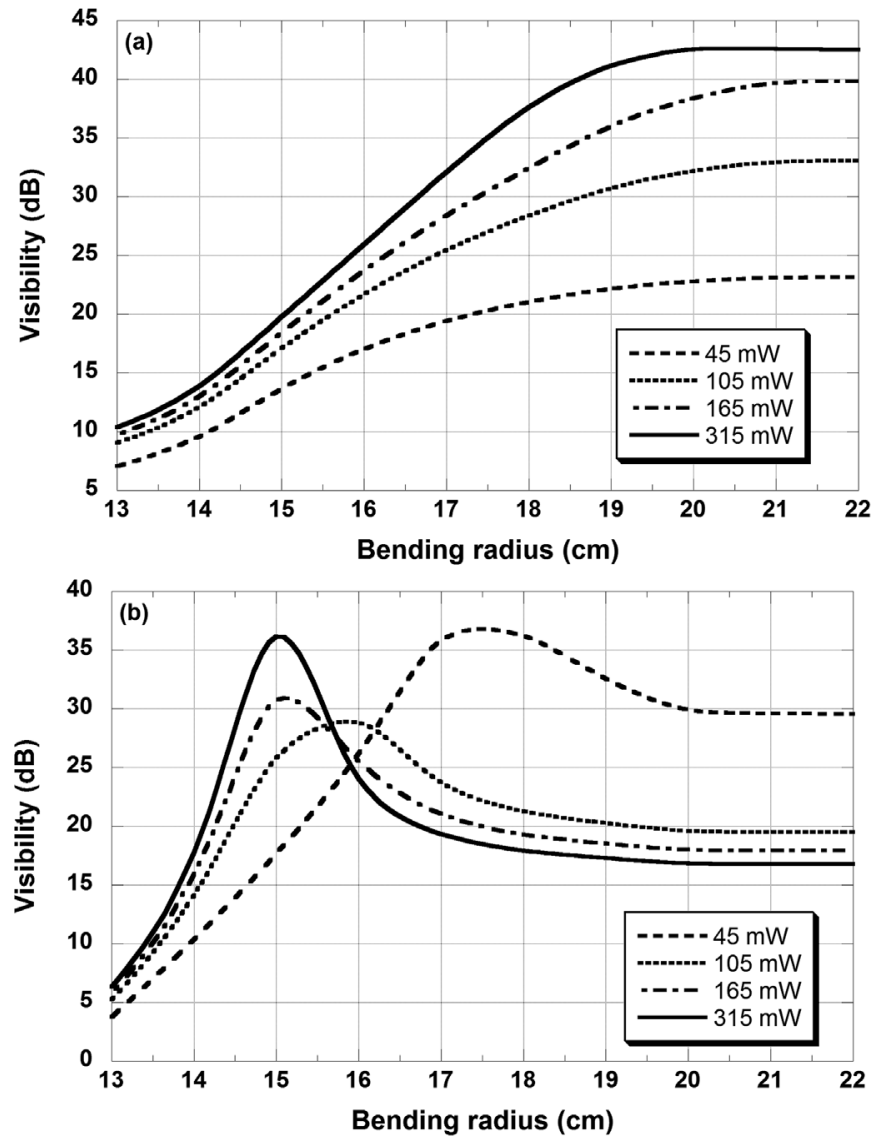

Fig. 7. Visibility as a function of the curvature radius used to calibrate the 7core MCF bending sensor active stage for transmission spectrum minimum at (a) $1524 \mathrm{~nm}$ and (b) $1544 \mathrm{~nm}$. Erbium doped ions concentration is $10^{26}$ ions/ $\mathrm{m}^{3}$, length is $10 \mathrm{~cm}$ and the different curves correspond to different pump powers.

$\frac{d P_{s, i}^{ \pm}\left(z, v_{s}\right)}{d z}= \pm\left[N_{2, s, i}(z) \sigma_{21}\left(v_{s}\right)-N_{1, s, i}(z) \sigma_{12}\left(v_{s}\right)-\alpha_{s, i}\right] P_{s, i}^{ \pm}\left(z, v_{s}\right)$

$$
\begin{aligned}
\frac{d P_{f, i}^{ \pm}\left(z, v_{f}\right)}{d z}= & \pm 2 h v \Delta v N_{2, s, i}(z) \sigma_{21}\left(v_{f}\right) \pm\left[N_{2, s, i}(z) \sigma_{21}\left(v_{f}\right)-N_{1, s, i}(z) \sigma_{12}\left(v_{f}\right)\right. \\
& \left.-\alpha_{s, i}\right] P_{f, i}^{ \pm}\left(z, v_{f}\right)
\end{aligned}
$$

where $N_{j, \gamma, i}$ is the overlapping integral of the modal distribution of the supermode $i$ and the population distribution of level $j$ over the area where the erbium ions are confined and $\sigma_{i, j}$ are the transition effective cross sections. Losses that are not related to the ion absorption (passive losses) are described by the coefficients $\alpha_{s}$ and $\alpha_{p}$. This model can be used to design optical amplifiers, in particular erbium-doped MCF, once the necessary input parameters are known. These input parameters must be obtained experimentally [11].

In order to calculate the total power at a certain wavelength one must compute the gain of each mode $g_{i}$, and take it into account when calculating the field expression that will be projected into the output coupled SMF. Due to the translational invariance of the fiber, it is possible to express the field in the separable form

$\boldsymbol{E}_{j}(x, y, z)=\boldsymbol{e}_{j}(x, y) \cdot \exp \left(i \beta_{j} z\right)$

where $\beta_{j}$ is the propagation constant of the $j$ th mode, and where the time dependence term been omitted. By computing the optical power amplification the gain amplitude coefficient $g(z)$ can be obtained and included in the previous expression,

$\boldsymbol{E}_{j}(x, y, z)=\sqrt{g_{j}(z)} \cdot \boldsymbol{e}_{j}(x, y) \cdot \exp \left(i \beta_{j} z\right)$
The total field at the end of the MCF, is the interference of both supermode fields with their corresponding gain amplitude coefficient.

\section{Results}

\subsection{Supermode gain}

Typical lengths of multicore fiber sensors go from 1 up to $10 \mathrm{~cm}$. For our analysis we select $3 \mathrm{MCF}$ lengths close to 1,5 and $10 \mathrm{~cm}$ that ensure a constructive interference between both supermodes at the fiber output end for a $1534 \mathrm{~nm}$ wavelength. Since we will use transmission spectra fringes visibility to evaluate the bending sensor performance, the respective nearest minimum transmission wavelengths at both sides of the gain peak $(1534 \mathrm{~nm})$ have to be determined for the selected fiber lengths. The three fiber lengths and the minimum transmission wavelengths are summarized in Table 1.

The active behavior of the MCF-based interferometer at $1534 \mathrm{~nm}$ for the 3 lengths in Table 1 and for three erbium ion concentrations is shown in Fig. 5. In Fig. 5(a) the gain difference between both excited supermodes, $\Delta g=g_{a^{-}} g_{f}$, is plotted as a function of the pump power for the three lengths with $\mathrm{n}_{\mathrm{Er}}=10^{26}$ ion $/ \mathrm{m}^{3}$ whereas in Fig. 5(b) it is plotted for three erbium ion concentrations and $L=10 \mathrm{~cm}$. From these figures, the dependence between the minimum bend radius and the necessary active multicore length and $\mathrm{Er}^{3+}$ ion concentration to compensate losses can be determined.

\subsection{Fringes visibility}

The visibility is evaluated as the rate between the signal power at $1534 \mathrm{~nm}$ (constructive interference- $\mathrm{P}_{\max }$ ) and at a transmission minimum wavelength (destructive interference- $\mathrm{P}_{\min }$ ). If expressed in decibels the visibility takes the form:

$V=10 \cdot \log _{10}\left(\frac{P_{\max }}{P_{\min }}\right)$

For the lower wavelength transmission minima in Table 1 outside the emission band of the $\mathrm{Er}^{3+}$ ion, it is not necessary to compute the optical powers active propagation and just to consider absorption attenuation and passive losses. However, for the other wavelengths each excited supermode gain has to be computed to properly evaluate the visibility. Then, taking into account the amplification experienced by each mode and the constructive or destructive modal interference at the fiber output we can calculate the active MCF visibility for each fiber length. Fig. 6 shows the visibility as a function of the curvature radius for the three chosen lengths. In Fig. 6(a) considering the transmission minima lower wavelengths in Table 1 and in Fig. 6(b) with the longer wavelength ones. Visibility depends on the pump power and, as the gain spectrum is not symmetric with respect to the peak, also depends on the measured minimum. A significant difference between the visibility depedences in Fig. 6(a) and (b) is appreciable. The $\mathrm{L}=10 \mathrm{~cm}$ MCF visibility in Fig. 6(a) presents a linear variation with bending radius, whereas in Fig. 6(b) it presents a maximum near $15 \mathrm{~cm}$. This peak in visibility can be attributed to the difference in supermode gain that could balance both modes powers and generate a very low $\mathrm{P}_{\min }$ when they destructively interfere.

In Fig. 7 we present potential calibration curves of the proposed MCF bending sensor device. The $\mathrm{Er}^{3+}$-ion concentration, available input pump power and MCF length set the minimum bending radius that could be measured. For given fiber length and available pump power the measured visibility will determine the corresponding curvature radius. In Fig. 7, the visibility is plotted for (a) $\lambda=1524$ and (b) $1544 \mathrm{~nm}$ for different pump powers in the case of a $10 \mathrm{~cm}$ MCF. Clearly, to use a transmission minimum wavelength outside the gain bandwidth avoids the existence of visibility maxima as a function of the bending radius and simplifies the use of the visibility curves as bending sensor 
references. This fact introduces a limitation in the practical active MCF length since for longer multicore fibers both nearest minima of transmission spectra centered in $1534 \mathrm{~nm}$ would experience supermode gain.

\section{Conclusions}

In this study we have explored the feasibility of turning the MCF in a supermode-interference based bending sensor into an active stage in order to improve fringe visibility for high curvatures and extend the sensor operating range. It is concluded that the $\mathrm{Er}^{3+}$-ion concentration, available input pump power and MCF length set the minimum bending radius that could be measured. For given doping profile and MCF length, the sensor calibration curves allow to assess the curvature radius by measuring the visibility as a function of the input pump power. The results of this numerical feasibility study have encouraged the authors to perform a coming experimental test to corroborate the active MCF bending sensor predicted performance.

This procedure could be implemented not only in fiber bending sensors but in all kind of interferometer-based fiber sensor where notable differences in modal losses can have a detrimental impact on the fringes visibility.

\section{Acknowledgements}

This work was partially supported by the Spanish Ministry of Economy and Competitiveness under the TEC2014-52642-C2 project, by the Diputación General de Aragón (DGA) and by the Fondo Social Europeo.

\section{References}

[1] J. Villatoro, E. Antonio-Lopez, A. Schülzgen, R. Amezcua-Correa, Miniature multicore optical fiber vibration sensor, Optic Lett. 42 (10) (2017) 2022-2025.

[2] A. Van Newkirk, E. Antonio-Lopez, G. Salceda-Delgado, R. Amezcua-Correa, A. Schülzgen, Optimization of multicore fiber for high-temperature sensing, Optic Lett. 39 (16) (2014) 4812-4815.

[3] G. Salceda-Delgado, A. Van Newkirk, J.E. Antonio-Lopez, A. Martinez-Rios, A. Schülzgen, R.A. Correa, Compact fiber-optic curvature sensor based on supermode interference in a seven-core fiber, Optic Lett. 40 (7) (2015) 1468-1471.

[4] G.P. Agrawal, Nonlinear Fiber Optics, Academic, San Diego, 1995, pp. 263-265 Google Scholar.

[5] W. Ren, Z. Tan, G. Ren, Analytical formulation of supermodes in multicore fibers with hexagonally distributed cores, IEEE Photonics Journal 7 (1) (2015) 1-11 [6] Cen Xia, Neng Bai, Ibrahim Ozdur, Xiang Zhou, and Guifang Li. Supermodes for optical transmission. Optics express, 19(17):16653\{16664, 2011.

[6] A.W. Snyder, J. Love, Optical Waveguide Theory, Springer Science \& Business Media, 2012

[7] J. Villatoro, A. Van Newkirk, E. Antonio-Lopez, J. Zubia, A. Schülzgen, R. AmezcuaCorrea, Ultrasensitive vector bending sensor based on multicore optical fiber, Optic Lett. 41 (4) (2016) 832-835.

[8] A. Van Newkirk, J.E. Antonio-Lopez, A. Velazquez-Benitez, J. Albert, R. AmezcuaCorrea, A. Schülzgen, Bending sensor combining multicore fiber with a mode-selective photonic lantern, Optic Lett. 40 (22) (2015) 5188-5191.

[9] E. Desurvire, Erbium-doped Fiber Amplifiers: Principles and Applications, WileyInterscience, 2002.

[10] J.A. Valles, V. Berdejo, M.Á. Rebolledo, A. Diez, J.A. Sanchez-Martin, M.V. Andres, Dynamic characterization of upconversion in highly Er-doped silica photonic crystal fibers, IEEE J. Quant. Electron. 48 (8) (2012) 1015-1022.

[11] J.A. Sánchez-Martín, M.Á. Rebolledo, J.M. Álvarez, J.A. Valles, A. Diez, M.V. Andres, Erbium-doped-silica photonic crystal fiber characterization method: description and experimental check, IEEE J. Quant. Electron. 46 (8) (2010) 1145-1152. 